\title{
On robust synchronization of nonlinear systems with application to grid integration of renewable energy sources
}

\author{
Hafiz Ahmed $^{\mathrm{a}, *}$, Rosane Ushirobira ${ }^{\mathrm{b}}$, Denis Efimov ${ }^{\mathrm{b}}$ \\ ${ }^{a}$ Nuclear Futures Institute, Bangor University, Bangor, LL57 1UT, United Kingdom. \\ ${ }^{b}$ Inria, Univ. Lille, CNRS, UMR 9189 - CRIStAL - Centre de Recherche en Informatique \\ Signal et Automatique de Lille, F-59000 Lille, France
}

\begin{abstract}
Many systems in the natural and physical world often work in unison with similar other systems. This process of simultaneous operation is known as synchronization. In the past few decades, owing to this phenomenon's importance, extensive research efforts have been made. However, many of the existing results consider the systems are identical and/or linear time-invariant, while practical systems are often nonlinear and nonidentical for various reasons. This observation motivated several recent studies on the synchronization of nonidentical (i.e., heterogeneous) nonlinear systems. This paper summarizes some recent results on the synchronization of heterogeneous nonlinear systems, as developed in the thesis [1]. First, the results on the synchronization of a particular class of robustly stable nonlinear systems are presented. Then, these results are applied to an example model known as Brockett oscillator. Finally, using the Brockett oscillator as a common dynamics, output oscillatory synchronization results are given for heterogeneous nonlinear systems of relative degree 2 or higher. An application example of Brockett oscillator for power-grid synchronization is also presented. Some outlooks are provided regarding future research directions.
\end{abstract}

Keywords: Robust Synchronization, Multistability, Output Synchronization, Oscillatory Synchronization, Renewable Energy Systems

\section{Contents}

1 Introduction 2

2 Preliminaries 4

2.1 Decomposable sets . . . . . . . . . . . . . . . 4

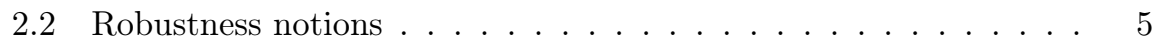

* Corresponding author

Email address: hafiz.h.ahmed@ieee.org (Hafiz Ahmed)

Preprint submitted to Annual Reviews in Control

April 15, 2021 
3 Synchronization of Nonlinear Systems $\quad 6$

3.1 Synchronization of robustly stable nonlinear systems . . . . . 6

3.2 Robust Synchronization of Brockett Oscillators . . . . . . . . 8

3.3 Oscillatory Output Synchronization of Heterogeneous Systems . . 10

3.3.1 Observer design . . . . . . . . . . . . . . . . 12

3.3.2 Synchronizing Controller Design _. . . . . . . . . 13

4 Grid-Synchronization of Renewable Energy Sources $\quad 15$

4.1 Experimental Study . . . . . . . . . . . . . 16

5 Conclusion and Outlook $\quad 17$

\section{Introduction}

Synchronization or collective behavior is omnipresent in natural and technological systems. It has motivated researchers across disciplines to scientifically investigate synchronization among a network of agents. These agents can be robots, cells in a tissue, human, birds, to name a few [2]. Synchronization is useful to ensure efficient operation of many technological systems such as power grid [3], synthetic genetic oscillator [4], cyber-attack resilient communication $[5,6]$, navigation through global positioning systems [7]. In many of these applications, synchronization essentially means the phase and frequency adjustment of oscillators. This highlights the importance of oscillator or oscillatory-type synchronization for natural and technological systems.

Owing to the enormous significance of synchronization, control systems researchers developed various control solutions to tackle synchronization. $\mathrm{Nu}-$ merous results have already been proposed and cover many areas of interest $[8,9,10,11]$. Initially, most of the results considered the case of synchronization among a network of identical agents that are linear time-invariant [12, 13]. In practice, engineering systems are often nonlinear, and it is very challenging to ensure that the systems are identical. Manufacturing defects, temperature, humidity, aging, and other factors often make two identical systems nonidentical over time, limiting the application of results based on the assumption of identical systems. In the heterogeneous systems case, i.e., when the agents are nonidentical, the network's behaviour becomes significantly difficult to analyze over the identical agents-based network counterpart. The difficulty arises from the lack of common equilibrium among the agents in the network.

Owing to the complicated nature of heterogeneous systems, the existing literature on the synchronization of these systems is limited. In general, it is difficult to obtain state synchronization for heterogeneous systems. So, some recent attempts have been made towards output synchronization, and these results rely on the idea of the internal model principle [14, 15, 16, 17]. These methods assign to each member of the network a local reference generator, and the synchronizing controllers work to track the reference by the system output. As the internal model is common to all network members, global information sharing is required among the agents. In addition to output synchronization, 


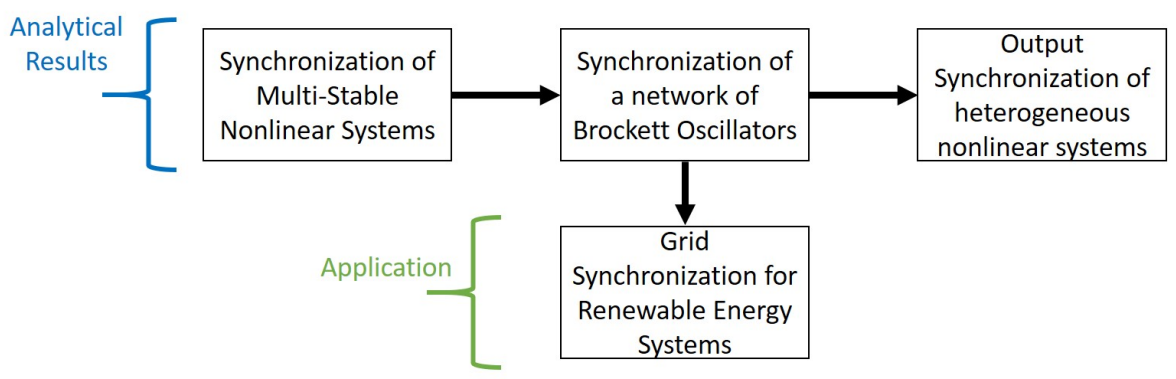

Figure 1: Overview of the results summarized in this paper.

some state synchronization approaches are also available in the literature. Moreover, many results are available for specific classes of systems such as passive ones $[18,19,20]$.

In this paper, our focus is to summarize the results presented in [1], where several methods on the robust synchronization of nonlinear systems are obtained. In the first instance, robust synchronization results for homogeneous or heterogeneous robustly stable nonlinear systems are considered, where the systems admit decomposition without cycles (neither homoclinic nor heteroclinic orbits) [21]. These results are then applied to a network of heterogeneous Brockett oscillators (BO) $[22,23,24,25]$ which are multi-stable. Using BO as the common dynamics, global output oscillatory synchronization results are proposed for heterogeneous nonlinear systems of relative degree 2 or higher [26]. An advantage of the BO-based approach is that it eliminates the need to share any global network information. Moreover, agents can have different dimensions and relative degrees, extending the types of systems that can be output-synchronized.

From a practical point of view, oscillator synchronization is critical in several engineering applications. Power-grid synchronization is a prominent real-world scenario of oscillator synchronization. The traditional power grid is based on alternating currents. Various energy sources are connected to this grid in a grid-following manner. So, oscillatory synchronization between the grid and the energy sources is essential for an efficient and smooth operation of the power grid. The BO model has recently been applied for grid-synchronization of renewable energy sources to the grid and grid-interfaced power electronic converters $[27,28,29,30]$. A summary of those approaches is also presented in this paper. An overview of the results compiled in this paper is given in Fig. 1.

The rest of the paper is organized as follows: Section 2 introduces some preliminaries about decomposable sets and notions of robustness. Section 3 presents the summary of the analytical results on the robust synchronization of heterogeneous nonlinear systems, Section 4 focuses on an application of a particular reference generator model presented in Section 3 to grid-synchronization of renewable energy sources, and finally, Section 5 provides a conclusion and outlook. 


\section{Preliminaries}

This section introduces some basic facts about decomposable sets and robustness notions as adapted from $[31,32]$. Let $M$ be an $n$-dimensional $\mathcal{C}^{2}$ connected and orientable Riemannian manifold without a boundary, $x \in M$ and $f: M \times \mathbb{R}^{m} \rightarrow T_{x} M$ be a map of class $\mathcal{C}^{1}$. In this work, we assume that all manifolds can be embedded in a Euclidean space of dimension $n$, so they contain 0 . Consider a nonlinear system of the following form:

$$
\dot{x}(t)=f(x(t), d(t))
$$

where the state $x(t) \in M$ and $d(t) \in \mathbb{R}^{m}$ for $t \geq 0$ (the input $d$ is a locally essentially bounded and measurable signal). For any initial condition $x_{0} \in M$ and input $d$, we denote by $X\left(t, x_{0} ; d\right)$ the uniquely defined solution of $(1)$ at time $t$ satisfying $X\left(0, x_{0} ; d\right)=x_{0}$. Together with (1), we will analyze its unperturbed version:

$$
\dot{x}(t)=f(x(t), 0) .
$$

A set $S \subset M$ is invariant for the unperturbed system (2) if $X\left(t, x_{0} ; 0\right) \in S$ for all $t \in \mathbb{R}$ and for all $x_{0} \in S$. For a set $S \subset M$, define the distance to $S$ from a point $x \in M$ by $|x|_{S}=\inf _{a \in S} \delta(x, a)$, where the $\delta\left(x_{1}, x_{2}\right)$ denotes the Riemannian distance between $x_{1}$ and $x_{2}$ in $M$. We have $|x|=|x|_{\{0\}}$ for $x \in M$, the usual Euclidean norm of a vector $x \in \mathbb{R}^{n}$. For a signal $d: \mathbb{R} \rightarrow \mathbb{R}^{m}$, the essential supremum norm is defined as $\|d\|_{\infty}=\operatorname{ess}_{\sup _{t>0}}|d(t)|$.

A function $\alpha: \mathbb{R}_{+} \rightarrow \mathbb{R}_{+}$is said to belong to class $\mathcal{K}($ or $\alpha \in \mathcal{K}$ ), if it is continuous, strictly increasing and $\alpha(0)=0$. Furthermore, $\alpha \in \mathcal{K}_{\infty}$ if $\alpha \in \mathcal{K}$ and unbounded, i.e., $\lim _{s \rightarrow+\infty} \alpha(s)=\infty$. For any $x \in M$, the $\alpha$ - and $\omega$ - limit sets for (2) can be defined as follows:

$$
\begin{aligned}
\alpha(x): & =\left\{y \in M \mid y=\lim _{n \rightarrow-\infty} X\left(x, t_{n}\right) \text { with } t_{n} \searrow-\infty\right\}, \\
\omega(x): & =\left\{y \in M \mid y=\lim _{n \rightarrow+\infty} X\left(x, t_{n}\right) \text { with } t_{n} \nearrow+\infty\right\} .
\end{aligned}
$$

\subsection{Decomposable sets}

Let $\Lambda \subset M$ be a compact invariant set for (2).

Definition 1. [33] $A$ decomposition of $\Lambda$ is a finite and disjoint family of compact invariant sets $\Lambda_{1}, \ldots, \Lambda_{k}$ such that $\Lambda=\bigcup_{i=1}^{k} \Lambda_{i}$.

For an invariant set $\Lambda$, its attracting and repulsing subsets are defined as follows:

$$
\begin{aligned}
W^{s}(\Lambda) & =\left\{\left.x \in M|| X(t, x, 0)\right|_{\Lambda} \rightarrow 0 \text { as } t \rightarrow+\infty\right\} \\
W^{u}(\Lambda) & =\left\{\left.x \in M|| X(t, x, 0)\right|_{\Lambda} \rightarrow 0 \text { as } t \rightarrow-\infty\right\} .
\end{aligned}
$$

Define a relation on the set of invariant sets of $M$ by: for $\mathcal{W} \subset M$ and $\mathcal{D} \subset M$, we write $\mathcal{W} \prec \mathcal{D}$ if $W^{s}(\mathcal{W}) \cap W^{u}(\mathcal{D}) \neq \emptyset$. 
Definition 2. [33] Let $\Lambda_{1}, \ldots, \Lambda_{k}$ be a decomposition of $\Lambda$, then

1. An $r$-cycle $(r \geq 2)$ is an ordered $r$-tuple of distinct indices $i_{1}, \ldots, i_{r}$ such that $\Lambda_{i_{1}} \prec \ldots \prec \Lambda_{i_{r}} \prec \Lambda_{i_{1}}$.

2. A 1-cycle is an index $i$ such that $\left(W^{u}\left(\Lambda_{i}\right) \cap W^{s}\left(\Lambda_{i}\right)\right) \backslash \Lambda_{i} \neq \emptyset$.

3. A filtration ordering is a numbering of the $\Lambda_{i}$ so that $\Lambda_{i} \prec \Lambda_{j} \Rightarrow i \leq j$.

As we can conclude from Definition 2, the existence of an $r$-cycle with $r \geq 2$ is equivalent to the existence of a heteroclinic cycle for (2) [34]. Moreover, the existence of a 1-cycle implies the existence of a homoclinic cycle for (2) [34].

Definition 3. Let $\mathcal{W} \subset M$ be a compact set containing all $\alpha$ and $\omega$ limit sets of (2). We say that $\mathcal{W}$ is decomposable if it admits a finite decomposition without cycles, $\mathcal{W}=\bigcup_{i=1}^{k} \mathcal{W}_{i}$, for some non-empty disjoint compact sets $\mathcal{W}_{i}$, forming a filtration ordering of $\mathcal{W}$.

This definition of the set $\mathcal{W}$ will be used all through the article.

\subsection{Robustness notions}

The following robustness notions for systems in (1) have been introduced in [31].

Definition 4. We say that the system (1) has the practical asymptotic gain ( $p A G)$ property if there exist $\eta \in \mathcal{K}_{\infty}$ and $q \in \mathbb{R}, q \geq 0$ such that for all $x \in M$ and all measurable essentially bounded inputs $d$, the solutions are defined for all $t \geq 0$ and

$$
\limsup _{t \rightarrow+\infty}|X(t, x ; d)|_{\mathcal{W}} \leq \eta\left(\|d\|_{\infty}\right)+q
$$

If $q=0$, then we say that the asymptotic gain ( $A G$ ) property holds.

Definition 5. We say that the system (1) has the limit property (LIM) with respect to $\mathcal{W}$ if there exists $\mu \in \mathcal{K}_{\infty}$ such that for all $x \in M$ and all measurable essentially bounded inputs $d$, the solutions are defined for all $t \geq 0$ and the following holds:

$$
\inf _{t \geq 0}|X(t, x ; d)| \mathcal{W} \leq \mu\left(\|d\|_{\infty}\right)
$$

Definition 6. We say that the system (1) has the practical global stability ( $p G S$ ) property with respect to $\mathcal{W}$ if there exist $\beta \in \mathcal{K}_{\infty}$ and $q \geq 0$ such that for all $x \in M$ and all measurable essentially bounded inputs $d(\cdot)$, the following holds for all $t \geq 0$ :

$$
|X(t, x ; d)|_{\mathcal{W}} \leq q+\beta\left(\max \left\{|x|_{\mathcal{W}},\|d\|_{\infty}\right\}\right)
$$

To characterize (3) in terms of Lyapunov functions, it has been shown in [31] that the following notion is suitable: 
Definition 7. We say that a $\mathcal{C}^{1}$ function $V: M \rightarrow \mathbb{R}$ is a practical ISSLyapunov function for (1) if there exists $\mathcal{K}_{\infty}$ functions $\alpha_{1},\left[\alpha_{2}\right], \alpha$ and $\gamma$, and scalar $q \geq 0$ [and $c \geq 0$ ] such that

$$
\alpha_{1}\left(|x|_{\mathcal{W}}\right) \leq V(x) \leq\left[\alpha_{2}\left(|x|_{\mathcal{W}}+c\right)\right],
$$

the function $V$ is constant on each $\mathcal{W}_{i}$ and the dissipation inequality below holds:

$$
D V(x) f(x, d) \leq-\alpha\left(|x|_{\mathcal{W}}\right)+\gamma(|d|)+q, \forall x \in M \text { and } d \in \mathbb{R}^{m} .
$$

If this latter holds for $q=0$, then $V$ is said to be an ISS-Lyapunov function.

Notice that $\alpha_{2}$ and $c$ are in square brackets as their existence follows (without any additional assumptions) by standard continuity arguments.

The main result of [31, Theorem 1, Page 3244] connecting these robust stability properties is stated below:

Theorem 1. Consider a nonlinear system as in (1) and let a compact invariant set containing all $\alpha$ - and $\omega$ - limit sets of (2) $\mathcal{W}$ be decomposable (in the sense of Definition 3). Then the following are equivalent:

1. The system admits an ISS Lyapunov function;

2. The system enjoys the AG property;

3. The system admits a practical ISS Lyapunov function;

4. The system enjoys the $p A G$ property;

5. The system enjoys the LIM property and the $p G S$.

A system in (1) that satisfies this list of equivalent properties is called ISS with respect to the set $\mathcal{W}$ [31].

\section{Synchronization of Nonlinear Systems}

\subsection{Synchronization of robustly stable nonlinear systems}

Let us consider a family of nonlinear systems for $i=1, \ldots, N>1$, as described by the following model:

$$
\dot{x}_{i}(t)=f_{i}\left(x_{i}(t), u_{i}(t), d_{i}(t)\right), \forall t \geq 0
$$

where the state $x_{i}(t) \in M_{i}\left(M_{i}\right.$ is $n_{i}$-dimensional $\mathcal{C}^{2}$ connected and orientable Riemannian manifold without a boundary), the control $u_{i}(t) \in \mathbb{R}^{m_{i}}$ and the external disturbance $d_{i}(t) \in \mathbb{R}^{p_{i}}\left(u_{i}\right.$ and $d_{i}$ are locally essentially bounded and measurable signals) for $t \geq 0$; and the map $f_{i}: M_{i} \times \mathbb{R}^{m_{i}} \times \mathbb{R}^{p_{i}} \rightarrow$ $T_{x_{i}} M_{i}$ is $\mathcal{C}^{1}, f_{i}(0,0,0)=0$. The common state vector of (4) is denoted as $x=\left[x_{1}^{T}, \ldots, x_{N}^{T}\right]^{T} \in M=\prod_{i=1}^{N} M_{i}$, so $M$ is the corresponding Riemannian manifold of dimension $n=\sum_{i=1}^{N} n_{i}$ where the family (4) evolves and $d=\left[d_{1}^{T}, \ldots, d_{N}^{T}\right]^{T} \in \mathbb{R}^{p}$ with $p=\sum_{i=1}^{N} p_{i}$ is the exogenous input. For the systems in (4), robust synchronization results have been provided in [1, 21] and recalled here. The results start with the robust stability assumption, which is given below: 
Assumption 1. Each family member in (4) (i.e. $\forall i=\overline{1, N}$ ) has a decomposable (as per Definition 3) compact invariant set $\mathcal{W}_{i}$ containing all $\alpha$ - and $\omega$-limit sets of $\dot{x}_{i}(t)=f_{i}\left(x_{i}(t), 0,0\right)$, and the system is input-to-state stable (ISS) with respect to the set $\mathcal{W}_{i}$ and the inputs $u_{i}$ and $d_{i}, \mathcal{W}=\mathcal{W}_{1} \times \cdots \times \mathcal{W}_{N}$.

A comprehensive review about ISS with respect to decomposable invariant sets can be found in [35].

To characterize synchronization for the systems in (4), the synchronization measure is considered by the function $\psi: M \rightarrow \mathbb{R}^{q}, \psi(0)=0$ which is a $\mathcal{C}^{1}$ function. Then, a formal definition of synchronization is given below:

Definition 8. For a suitably designed control signal u, systems in (4) will reach a consensus (being synchronized) if $\psi(x(t)) \equiv 0$ for all $t \geq 0$ on the solutions of the network for $d(t) \equiv 0, t \geq 0$. If, additionally, for bounded $d \neq \equiv 0$, the synchronization measure $\psi$ stays bounded, then the family (4) is said to be robustly synchronized.

The synchronizing control law is given by:

$$
u_{i}(t)=\varphi_{i}(\psi(x(t))), \forall t \geq 0
$$

$\left(\varphi_{i}: \mathbb{R}^{q} \rightarrow \mathbb{R}^{m_{i}}\right.$ is a $\mathcal{C}^{1}$ function, $\left.\varphi_{i}(0)=0\right)$. In the synchronous state, the set $\mathcal{A}=\{x \in \mathcal{W} \mid \psi(x)=0\}$ contains the synchronous solutions of the unperturbed systems in (4) and the problem of synchronization of "natural" trajectories is considered since $\mathcal{A} \subset \mathcal{W}$ (due to $\varphi_{i}(0)=0$ in (5), the convergence of $\psi$ (synchronization/consensus) implies that the solutions belong to $\mathcal{W}$ ).

For the systems in (4), a kind of robust synchronization with respect to any measurable and essentially bounded external disturbance $d$ is possible by suitably selecting the shape of each function $\varphi_{i}$. This is the main result of [21] and a summary is given below:

Proposition 1. Let Assumption 1 be satisfied for systems (4). Then, there exist functions $\varphi_{i}, i=\overline{1, N}$, for individual controllers (5) such that the closedloop system (4), (5) has the pGS property (as per Definition 7) with respect to the set $\mathcal{W}$.

For any suitably bounded function $\varphi_{i}$, in (5), the result of Proposition 1 is valid.

Assumption 2. The set $\mathcal{A}$ is compact, and it contains all $\alpha$-and $\omega$-limit sets of the closed-loop system (i.e., systems (4) and controllers (5)) for $d \equiv 0$, and it is decomposable.

Here additionally, it is assumed that the controls $\varphi_{i}$ ensure the network global synchronization in the noise-free case, while the decomposability, in general, follows from Assumption 1.

Theorem 2. Let conditions of Proposition 1 be satisfied together with Assumption 2. Then the closed-loop system (i.e., systems (4) and controllers (5)) is ISS with respect to $\mathcal{A}$. 


\subsection{Robust Synchronization of Brockett Oscillators}

In the previous section, an approach for robust synchronization of multistable systems is reviewed. Limit cycle oscillators exhibit multistability. R. Brockett recently studied the synchronization problem for BO in such a context $[23]^{1}$. However, the results presented in [23] can only guarantee local synchronization. The results presented here, though, provide global synchronization results for BO. For this purpose, the following family of BO is considered in this section for $i=\overline{1, N}$ and some $N>1$ :

$$
\begin{aligned}
& \dot{x}_{1 i}=x_{2 i}, \\
& \dot{x}_{2 i}=a_{i} u_{i}-x_{1 i}-b_{i} x_{2 i}\left(\left|x_{i}\right|^{2}-1\right),
\end{aligned}
$$

where the positive model parameters of individual $\mathrm{BO}$ are given by $a_{i}, b_{i}>0$, the state of individual BO is given by $x_{i}=\left[\begin{array}{ll}x_{1 i} & x_{2 i}\end{array}\right]^{T} \in \mathbb{R}^{2}$ and the control input for individual BO is denoted by $u_{i} \in \mathbb{R}\left(u_{i}: \mathbb{R}_{+} \rightarrow \mathbb{R}\right.$ and has the same property of input function as for the systems (4)). Similar to systems (4), $x=\left[x_{1}^{T}, \ldots, x_{N}^{T}\right]^{T} \in \mathbb{R}^{2 N}$ and $u=\left[u_{1}, \ldots, u_{N}\right]^{T} \in \mathbb{R}^{N}$ denote the common system state and control input vector for the network of BO (6).

The control input vector for the network (6) is chosen as:

$$
u=k \psi(x)=k \Pi \bar{x}_{2},
$$

where the positive constant $k$ denotes the network coupling gain and

$$
\Pi=\left[\begin{array}{ccccc}
-2 & 1 & 0 & \cdots & 1 \\
1 & -2 & 1 & \cdots & 0 \\
0 & 1 & -2 & \cdots & 0 \\
\vdots & & \vdots & \ddots & 0 \\
1 & \cdots & 0 & 1 & -2
\end{array}\right], \bar{x}_{2}=\left[\begin{array}{c}
x_{21} \\
\vdots \\
x_{2(N-1)} \\
x_{2 N}
\end{array}\right] .
$$

In (7), $\Pi$ is the interconnection matrix that describes the information sharing arrangement among the oscillators. The interconnection matrix $\Pi$ is considered by assuming that the oscillators are connected using an $N$-cycle graph. In this setting, each member of the network (6) requires only the information of the left and right neighbor oscillator. Let us define the synchronization error among the oscillators as:

$$
e_{2 i-1}=x_{1 i}-x_{1(i+1)}, \dot{e}_{2 i-1}=x_{2 i}-x_{2(i+1)}=e_{2 i}
$$

and $e_{2 N-1}=x_{1 N}-x_{11}, \dot{e}_{2 N-1}=x_{2 N}-x_{21}=e_{2 N}$. Then the main results of [22] can be summarized as below:

\footnotetext{
${ }^{1}$ It is to be noted here that the model considered by Brockett can also be found in [2] under the name circular limit cycle oscillator.
} 
Proposition 2. [22, Proposition 11, Page 294] All trajectories of the closedloop system (6), (7) are bounded and converge to the largest invariant set in

$$
\begin{aligned}
& \Omega_{\infty}=\left\{x \in \mathbb{R}^{2 N}:\left|x_{i}\right|=\text { const, } e_{2 i-1}^{2}+e_{2 i}^{2}=\right.\text { const } \\
& \left.x_{2(i-1)}+x_{2(i+1)}=\left(2+\frac{b_{i}}{a_{i} k}\left(\left|x_{i}\right|^{2}-1\right)\right) x_{2 i}, i=\overline{1, N}\right\}
\end{aligned}
$$

for any positive network coupling gain $k>0$.

Theorem 3. [22, Theorem 12, Page 294] If there is at least one BO with index $1 \leq i \leq N$ for which $2 a_{i} k<b_{i}$, then all trajectories of the closed-loop system (6), (7) are bounded and almost all of them converge to the largest invariant set in

$$
\begin{gathered}
\Omega_{\infty}^{\prime}=\left\{x \in \mathbb{R}^{2 N}:\left|x_{i}\right|=\mathrm{const} \neq 0, e_{2 i-1}^{2}+e_{2 i}^{2}=\mathrm{const}\right. \\
\left.x_{2(i-1)}+x_{2(i+1)}=\left(2+\frac{b_{i}}{a_{i} k}\left(\left|x_{i}\right|^{2}-1\right)\right) x_{2 i}, i=\overline{1, N}\right\}
\end{gathered}
$$

Proof. Since all conditions of Proposition 2 are satisfied, then all trajectories converge to the set $\Omega_{\infty}$. By substitution of the control (7) in the equations of (6) we obtain:

$$
\begin{aligned}
\dot{x}_{1 i}= & x_{2 i}, \\
\dot{x}_{2 i}= & a_{i} u_{i}-x_{1 i}-b_{i} x_{2 i}\left(\left|x_{i}\right|^{2}-1\right) \\
= & a_{i} k\left(x_{2(i-1)}-2 x_{2 i}+x_{2(i+1)}\right)-x_{1 i} \\
& -b_{i} x_{2 i}\left(\left|x_{i}\right|^{2}-1\right) \\
= & -x_{1 i}-\left(2 a_{i} k-b_{i}\right) x_{2 i}+a_{i} k\left(x_{2(i-1)}\right. \\
& \left.+x_{2(i+1)}\right)-b_{i} x_{2 i}\left|x_{i}\right|^{2} .
\end{aligned}
$$

Linearizing this system around the origin $\left(\left|x_{i}\right|=0\right.$ for all $\left.i=\overline{1, N}\right)$ we conclude that this equilibrium is unstable if there exists at least one index $1 \leq i \leq N$ with $2 a_{i} k<b_{i}$. Thus, for almost all initial conditions, the trajectories converge to a subset of $\Omega_{\infty}$ where $\left|x_{i}\right| \neq 0$, i.e. to the set $\Omega_{\infty}^{\prime}$ (see Proposition 11 in $[36])$.

This theorem establishes conditions for the existence of various oscillatory synchronization patterns in (6), (7). However, additional conditions need to be checked to guarantee the desired synchronization pattern with $\psi(x(t))=0$.

In the set $\Omega_{\infty}$, we have for all $i=\overline{1, N}$ :

$$
\begin{gathered}
x_{1 i}^{2}+x_{2 i}^{2}=r_{i}^{2}, \\
\rho_{i}^{2}=e_{2 i-1}^{2}+e_{2 i}^{2}=r_{i}^{2}+r_{i+1}^{2}-2\left(x_{1 i} x_{1(i+1)}+x_{2 i} x_{2(i+1)}\right)
\end{gathered}
$$

for some $r_{i} \in \mathbb{R}_{+}$and $\rho_{i} \in \mathbb{R}_{+}$, and

$$
x_{2(i-1)}+x_{2(i+1)}=\beta_{i} x_{2 i}, x_{1(i-1)}+x_{1(i+1)}=\beta_{i} x_{1 i}+c_{i}
$$

for $\beta_{i}=2+\alpha_{i}\left(r_{i}^{2}-1\right), \alpha_{i}=\frac{b_{i}}{k a_{i}}$ and some $c_{i} \in \mathbb{R}$. 
Corollary 1. [22, Corollary 13, Page 296] Let all conditions of Theorem 3 be satisfied, and all solutions of the following equations

$$
\begin{aligned}
\rho_{i}^{2}= & \frac{1+\alpha_{i}\left(r_{i}^{2}-1\right)}{2+\alpha_{i}\left(r_{i}^{2}-1\right)} r_{i+1}^{2}-\left(1+\alpha_{i}\left(r_{i}^{2}-1\right)\right) r_{i}^{2} \\
& +\frac{1}{2+\alpha_{i}\left(r_{i}^{2}-1\right)} r_{i-1}^{2}, i=\overline{1, N} \\
0= & \sum_{i=1}^{N}\left(\rho_{i}^{2}-r_{i}^{2}-r_{i+1}^{2}\right) k\left(a_{i}+a_{i+1}\right) \\
& +2 r_{i}^{2}\left(b_{i}\left(r_{i}^{2}-1\right)+2 k a_{i}\right)
\end{aligned}
$$

with $r_{i} \neq 1$ admit the restriction:

$$
r_{i}^{2}<\frac{1}{3}\left(1-2 \frac{k a_{i}}{b_{i}}\right)
$$

for some $1 \leq i \leq N$. Then for almost all initial conditions, the closed-loop system (i.e., system (6) and (7)) has reached consensus (i.e., synchronized).

Here (9) and (10) form the system of $N+1$ nonlinear algebraic equations for $2 N$ unknowns $\left(r_{i}\right.$ and $\rho_{i}$ ) describing the kind of synchronization in (6), (7) that is admissible in $\Omega_{\infty}^{\prime}((10)$ is not a linear combination of (9)). As we may note, $b_{i}>2 a_{i} k$ is a necessary condition for Corollary 1 to satisfy.

\subsection{Oscillatory Output Synchronization of Heterogeneous Systems}

The previous subsections present a generic framework for robust synchronization of multistable systems. However, in subsection 3.2, it is required that the individual subsystems are of the same order, which can be limiting in practice. Hence, to overcome this limitation, the results of [21, 22] are applied in [26] to a more general class of systems. Oscillatory output synchronization results are achieved among a network of heterogeneous nonlinear systems that satisfy certain conditions regarding the relative degree of the system by using only output feedback. As only output feedback is considered, a nonlinear observer is first applied to estimate the unmeasurable states and perturbations of individual systems. Then, through feedback transformation, nonlinearities are compensated, and the dynamics of the $\mathrm{BO}$ is added to convert part of the individual systems to a BO. Then, the global synchronization results of $\mathrm{BO}$ are applied to provide global oscillatory output synchronization among the heterogeneous nonlinear systems. Since the synchronization of BO is applied, individual systems must have at least the same relative degree (i.e., 2) of BO. For the definition of relative degree, interested readers can consult [37]. It is to be noted here that many typical engineering systems do satisfy the relative degree condition if an appropriate output is chosen. 
Let us consider control-affine single-input single-output systems in normal forms with globally defined relative degrees [37] for $i=\overline{1, N}, N>1$ :

$$
\begin{aligned}
\dot{\eta}_{i} & =\varsigma_{i}\left(\eta_{i}, \xi_{i}\right) \\
\dot{\xi}_{i} & =A_{r_{i}} \xi_{i}+b_{r_{i}}\left[\alpha_{i}\left(\xi_{i}\right)+\beta_{i}\left(\xi_{i}\right) u_{i}\right], \\
y_{i} & =c_{r_{i}} \xi_{i},
\end{aligned}
$$

where the state and input are denoted by $x_{i}=\left[\eta_{i}^{T} \xi_{i}^{T}\right]^{T} \in \mathbb{R}^{n_{i}}, u_{i} \in \mathbb{R}, \xi_{i} \in \mathbb{R}^{r_{i}}$ and $\eta_{i} \in \mathbb{R}^{n_{i}-r_{i}}$ are components of the state; $y_{i} \in \mathbb{R}$ is the output; $\varsigma_{i}: \mathbb{R}^{n_{i}} \rightarrow$ $\mathbb{R}^{n_{i}-r_{i}}, \alpha_{i}: \mathbb{R}^{r_{i}} \rightarrow \mathbb{R}$ and $\beta_{i}: \mathbb{R}^{r_{i}} \rightarrow \mathbb{R}$ are smooth functions, $r_{i} \in\left[2, n_{i}\right]$ is the relative degree, $\beta_{i}$ is separated from zero, and

$$
\begin{gathered}
A_{r_{i}}=\left[\begin{array}{cccc}
0 & 1 & 0 \ldots 0 & 0 \\
0 & 0 & 1 \ldots 0 & 0 \\
\vdots & \vdots & \ddots & \vdots \\
0 & 0 & 0 \ldots 0 & 1 \\
0 & 0 & 0 \ldots 0 & 0
\end{array}\right], b_{r_{i}}=\left[\begin{array}{c}
0 \\
0 \\
\vdots \\
0 \\
1
\end{array}\right], \\
c_{r_{i}}=\left[\begin{array}{ll}
1 & 0 \ldots 0
\end{array}\right]
\end{gathered}
$$

are in the canonical form. Moreover, $x=\left[x_{1}^{T}, \ldots, x_{N}^{T}\right]^{T} \in \mathbb{R}^{n}$ with $n=\sum_{i=1}^{N} n_{i}$, $y=\left[y_{1}, \ldots, y_{N}\right]^{T} \in \mathbb{R}^{N}, u=\left[u_{1}, \ldots, u_{N}\right]^{T} \in \mathbb{R}^{N}$ denote the common state, output, and input vector, respectively. The conditions of existence of normal form and the definition of relative degree can be found in [37].

The subsystem (12) is called the zero dynamics of $i^{\text {th }}$ subsystem, which we assume to be robustly stable:

Assumption 3. For all $i=\overline{1, N}$, the systems in (12) are ISS with respect to the inputs $\xi_{i}[38]$.

Definition 9. [26, Definition 2.1, Page 3] The family (12),(13) exhibits a global output synchronization if

$$
\lim _{t \rightarrow \infty}\left(y_{i}(t)-y_{j}(t)\right)=0, \quad \forall i, j=\overline{1, N}
$$

for any initial conditions $x_{i}(0) \in \mathbb{R}^{n_{i}}, i=\overline{1, N}$.

Note that under the robust stability of the zero dynamics (12), an additional requirement can be imposed on synchronization of derivatives:

$$
\lim _{t \rightarrow \infty}\left(\dot{y}_{i}(t)-\dot{y}_{j}(t)\right)=0 \quad \forall i, j=\overline{1, N}
$$

and an auxiliary restriction for synchronization is

$$
y_{i} \not \equiv \text { constant }, \quad \forall i=\overline{1, N},
$$

i.e., the systems perform some oscillations in the synchronous mode. 
To achieve the global oscillatory output synchronization for (13), a suitable output-feedback based controller needs to be designed. To facilitate this, one can simplify the second term in (13) by introducing a disturbance $d_{i} \in \mathbb{R}, i=\overline{1, N}$, as follows:

$$
u_{i}+d_{i}=\alpha_{i}\left(\xi_{i}\right)+\beta_{i}\left(\xi_{i}\right) u_{i} .
$$

As the functions $\beta_{i}$ are not singular, such a simplification always exists. Derivative boundedness of the newly introduced disturbance term is formally assumed:

Assumption 4. For all $i=\overline{1, N}$, the unknown input $d_{i}: \mathbb{R}_{+} \rightarrow \mathbb{R}$ is continuously differentiable for almost all $t \geq 0$, and there is a constant $0<\nu^{+}<+\infty$ such that ess $\sup _{t>0}\left|\dot{d}_{i}(t)\right| \leq \nu^{+}$.

\subsubsection{Observer design}

To compensate for the individual nonlinearities and disturbances of individual systems in (13), they need to be separated and estimated. For this purpose, the ideas presented in [39] and [40] will be used, and a Luenberger observer is utilized to decouple the aligned control and disturbance inputs. Let us consider that $\zeta_{i} \in \mathbb{R}^{r_{i}}$ is the Luenberger observer-based estimation of $\xi_{i}$, and the suitably designed observer gain is $l_{i} \in \mathbb{R}^{r_{i}}$, which ensures that the closed-loop error matrix is Hurwitz. The Luenberger observer is given by:

$$
\dot{\zeta}_{i}=A_{r_{i}} \zeta_{i}+b_{r_{i}} u_{i}+l_{i}\left(y_{i}-c_{r_{i}} \zeta_{i}\right),
$$

Then, the error dynamics for the extended error vector is given by:

$$
\dot{\tilde{e}}_{i}=A_{r_{i}+1} \widetilde{e}_{i}-\widetilde{l}_{i} \varepsilon_{i}+b_{r_{i}+1} \dot{d}_{i}
$$

where extended error vector $\widetilde{e}_{i}=\left[\begin{array}{ll}e_{i}^{T} & d_{i}\end{array}\right]^{T}, e_{i}=\xi_{i}-\zeta_{i}, \varepsilon_{i}=c_{r_{i}} e_{i}$, and $\widetilde{l}_{i}=$ $\left[\begin{array}{ll}l_{i}^{T} & 0\end{array}\right]^{T}$. The following high order sliding mode (HOSM) differentiator is applied to estimate the extended error vector $\tilde{e}_{i}$ :

$$
\begin{gathered}
\dot{z}_{i, 1}=\nu_{i, 1}=-\lambda_{i, 1}\left|z_{i, 1}-\varepsilon_{i}\right|^{\frac{r_{i}}{r_{i}+1}} \operatorname{sign}\left(z_{i, 1}-\varepsilon_{i}\right) \\
+z_{i, 2}-\widetilde{l}_{i, 1} \varepsilon_{i} \\
\dot{z}_{i, j}=\nu_{i, j}=-\lambda_{i, j}\left|z_{i, j}-\nu_{i, j-1}\right|^{\frac{r_{i}-j+1}{r_{i}-j+2}} \operatorname{sign}\left(z_{i, j}-\nu_{i, j-1}\right) \\
+z_{i, j+1}-\widetilde{l}_{i, j} \varepsilon_{i}, j=\overline{2, r_{i}} \\
\dot{z}_{i, r_{i}+1}=-\lambda_{i, r_{i}+1} \operatorname{sign}\left(z_{i, r_{i}+1}-\nu_{i, r_{i}}\right)
\end{gathered}
$$

where $\lambda_{i}=\left[\lambda_{i, 1} \ldots \lambda_{i, r_{i}+1}\right]^{T} \in \mathbb{R}^{r_{i}+1}$ is the vector of HOSM differentiator tuning gains. The closed-loop observer system, i.e., systems (12), (13) and observers (14), (15), is discontinuous and its solutions are to be understood in the Filippov sense [41]. By combining the outputs of the Luenberger observer (14) and the 
HOSM differentiator (15), states $\xi_{i}$ and disturbances $d_{i}$ of individual systems can be estimated as:

$$
\widehat{\xi}_{i}=\zeta_{i}+\left[\begin{array}{c}
z_{i, 1} \\
\vdots \\
z_{i, r_{i}}
\end{array}\right], \widehat{d}_{i}=z_{i, r_{i}+1}
$$

Finite-time stability of the estimation errors for state and disturbances is proven in [26, Proposition 3.1, Page 4]:

Proposition 3. Let Assumption 4 be satisfied, the matrices $A_{r_{i}}-l_{i} c_{r_{i}}$ be Hurwitz. Then there exist $\lambda_{i} \in \mathbb{R}^{r_{i}+1}\left(\lambda_{i, r_{i}+1}>\nu^{+}\right.$for all $\left.i=\overline{1, N}\right)$ and $\mathcal{T}_{i}>0$ such that for the system in (13) and the observer in (14), (15), for all $t \geq \mathcal{T}_{i}$ :

$$
\begin{gathered}
y_{i}^{(j)}(t)=\widehat{\xi}_{i, j}(t), j=\overline{1, r_{i}} \\
d_{i}(t)=\widehat{d}_{i}(t)
\end{gathered}
$$

\subsubsection{Synchronizing Controller Design}

Two controllers are designed in [26] for systems of relative degree 2 and/or higher. These controllers are summarised below:

The relative degree 2 case. In this case, $r_{i}=2$. The synchronization controllers are designed for all $i=\overline{1, N}$ in the following form:

$$
\begin{aligned}
u_{i} & =\underbrace{-\widehat{d}_{i}}_{\text {part } 1} \underbrace{-\widehat{\xi}_{i, 1}-b_{i} \widehat{\xi}_{i, 2}\left(\widehat{\xi}_{i, 1}^{2}+\widehat{\xi}_{i, 2}^{2}-1\right)}_{\text {part } 2}+ \\
& +\underbrace{a_{i} k\left(\widehat{\xi}_{i-1,2}-2 \widehat{\xi}_{i, 2}+\widehat{\xi}_{i+1,2}\right)}_{\text {part } 3},
\end{aligned}
$$

where $a_{i}>0, b_{i}>0$ and $k_{i}>0$ are controller tuning parameters. First, the individual disturbance is compensated by part 1 in (16). Then, the dynamics of BO is injected by part 2 in (16). Finally, the synchronization is achieved by part 3 in (16). This controller is completely distributed as only the output of left and right neighbor is needed. Moreover, the observers (14) and (15) are also distributed. This makes the closed-loop system distributed.

Then, the main result of [26], for $r_{i}=2$, is given below:

Theorem 4. [26, Theorem 3.2, Page 4] Let assumptions 3 and 4 be satisfied, the matrices $A_{r_{i}}-l_{i} c_{r_{i}}$ be Hurwitz, $r_{i}=2$ and $\lambda_{i} \in \mathbb{R}^{r_{i}+1}$ for all $i=\overline{1, N}$ be selected as in Proposition 3. Consider the system (12),(13) with the observers (14), (15) and the synchronizing feedback control (16). If there is an index $1 \leq i \leq N$ such that $2 a_{i} k<b_{i}$, then all trajectories in the closed-loop system are bounded, and for almost all initial conditions, they converge to the largest invariant set, where the following restrictions are satisfied for all $i=\overline{1, N}$ : 


$$
\begin{gathered}
\dot{y}_{i-1}+\dot{y}_{i+1}=\left(2+\frac{b_{i}}{a_{i} k}\left(y_{i}^{2}+\dot{y}_{i}^{2}-1\right)\right) \dot{y}_{i}, \\
y_{i}^{2}+\dot{y}_{i}^{2}=\text { constant } \neq 0, \\
\left(y_{i}-y_{i+1}\right)^{2}+\left(\dot{y}_{i}-\dot{y}_{i+1}\right)^{2}=\text { constant } .
\end{gathered}
$$

The higher relative degree case. In the general case, i.e., $r_{i} \geq 2$, parts 2 and 3 of the control (16) form a reference signal $\xi_{i, 3}^{d}$ for the variable $\xi_{i, 3}$ :

$$
\begin{aligned}
\xi_{i, 3}^{d}= & -\widehat{\xi}_{i, 1}-b_{i} \widehat{\xi}_{i, 2}\left(\widehat{\xi}_{i, 1}^{2}+\widehat{\xi}_{i, 2}^{2}-1\right) \\
& +a_{i} k\left(\widehat{\xi}_{i-1,2}-2 \widehat{\xi}_{i, 2}+\widehat{\xi}_{i+1,2}\right)
\end{aligned}
$$

where the parameters $a_{i}>0, b_{i}>0$, and $k_{i}>0$ save their meaning, and next, this reference signal has to be propagated over chain of integrators, and part 1 of the control (16) has to be applied in the last step to annihilate $d_{i}$. Let us denote

$$
\tilde{\xi}_{i, s}=\xi_{i, s}-\xi_{i, s}^{d}
$$

as the error or realization of a desired signal $\widehat{\xi}_{i, s}^{d}$ by $\xi_{i, s}$ for $s=\overline{3, r_{i}}$. Then, by applying the backstepping-based design approach [42], the following controller has been designed in [26]:

$$
u_{i}=-\widehat{d}_{i}+\dot{\xi}_{i, r_{i}}^{d}-\kappa \tilde{\xi}_{i, r_{i}}-\tilde{\xi}_{i, r_{i}-1}
$$

The control (18) involves derivatives. These derivatives can be estimated by the HOSM differentiator similar to the one in (15) for $a=-1,+1$ :

$$
\begin{gathered}
\dot{\varrho}_{i, 0}^{a}=\nu_{i, 0}=-\mu_{i, 0}\left|\varrho_{i, 0}^{a}-\widehat{\xi}_{i+a, 2}\right|^{\frac{r_{i}-3}{r_{i}-2}} \operatorname{sign}\left(\varrho_{i, 0}^{a}-\widehat{\xi}_{i+a, 2}\right)+\varrho_{i, 1}^{a}, \\
\dot{\varrho}_{i, j}^{a}=\nu_{i, j}=-\mu_{i, j}\left|\varrho_{i, j}^{a}-\nu_{i, j-1}\right|^{\frac{r_{i}-j-3}{r_{i}-j-2}} \operatorname{sign}\left(\varrho_{i, j}^{a}-\nu_{i, j-1}\right) \\
+\varrho_{i, j+1}^{a}, j=\overline{1, r_{i}-4} \\
\dot{\varrho}_{i, r_{i}-3}^{a}=-\mu_{i, r_{i}-3} \operatorname{sign}\left(\varrho_{i, r_{i}-3}^{a}-\nu_{i, r_{i}}\right) .
\end{gathered}
$$

Then, by Lemma 8 in [40], for a proper selection of $\mu_{i}=\left[\mu_{i, 0}, \ldots, \mu_{i, r_{i}-3}\right]^{T}>0$ there is $\mathbb{T}_{i}>0$ such that

$$
\varrho_{i, j}^{a}(t)=\widehat{\xi}_{i+a, 2}^{(j)}(t), \quad \forall t \geq \mathbb{T}_{i}
$$

for all $j=\overline{0, r_{i}-3}$ and $a=-1,+1$. Note that from $(15), \widehat{\xi}_{i+a, 2}$ has only $r_{i+a}-1$ continuous derivatives, consequently

$$
r_{i} \leq r_{i+a}+2 \quad \forall i=\overline{1, N}, a=-1,+1 .
$$

The following result has been proven [26, Theorem 3.4, Page 6]: 


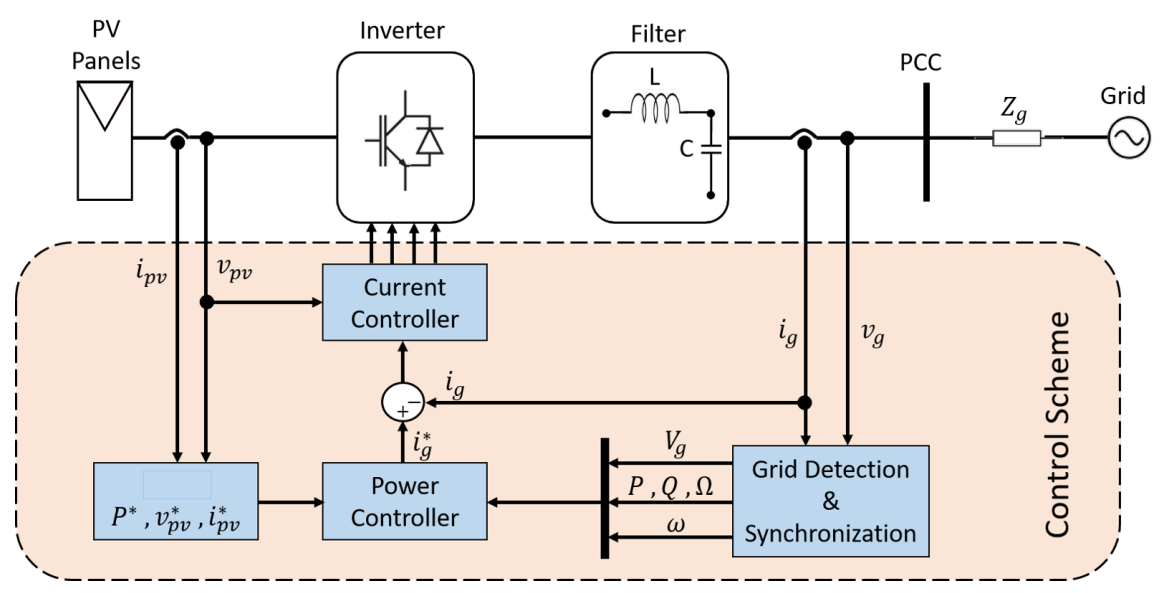

Figure 2: Control system overview of a grid-connected solar PV system.

Theorem 5. Let assumptions 3 and 4 be satisfied, the matrices $A_{r_{i}}-l_{i} c_{r_{i}}$ be Hurwitz, $r_{i} \geq 2$ under (20), and $\lambda_{i} \in \mathbb{R}^{r_{i}+1}, \mu_{i} \in \mathbb{R}^{r_{i}-2}$ be properly selected for all $i=\overline{1, N}$. Consider the system (12),(13) with the observers (14), (15), (19) and the synchronizing feedback control (18). If there is an index $1 \leq i \leq N$ such that $2 a_{i} k<b_{i}$, then all trajectories in the closed-loop system are bounded, and for almost all initial conditions they converge to the largest invariant set, where the restrictions (17) are satisfied.

\section{Grid-Synchronization of Renewable Energy Sources}

Renewable energy sources (RES) such as solar photovoltaics (PV) will play a vital role in achieving net-zero carbon emissions by 2050. RES are connected to the existing alternating current $(\mathrm{AC})$ grid through a voltage source inverter (VSI) [43, 44, 45]. An efficient operation of the RES-interfaced VSI can only be guaranteed when the output of the VSI matches in-phase with the grid voltage signal. This matching operation is known as grid-synchronization. Gridsynchronization demands real-time estimation of the grid phase so that the current controller can ensure in-phase operation of the VSI with the grid. An overview of the grid-connected solar PV control system is given in Fig. 2. In this section, a summary of the BO-based grid-synchronization technique is presented. For further consideration, the model of the single-phase grid voltage signal with offset is given by:

$$
v(t)=A_{0}+A \sin (\omega t+\phi),
$$

where the DC offset, amplitude, angular frequency and initial phase angle are denoted by $A_{0}, A, \omega$, and $\phi$, respectively. The instantaneous phase is often used to characterize the grid voltage signal and given by $\Omega=\omega t+\phi$ and $\Omega \in[0,2 \pi)$. The grid frequency $\omega$ is unknown but evolves around a known nominal value 
and the amplitude $A$ is conventionally normalized to 1 . Grid codes typically require the frequency to be within a certain band. The current controller of RES requires information $\Omega$ in real-time. This information can easily be obtained if an orthogonal signal of the grid-voltage (21) in real-time can be generated. BO can be used for this purpose, but $\mathrm{BO}$ are not frequency-adaptive as introduced in Section 3.2. To make BO frequency-adaptive, the equation (6) can be rewritten as:

$$
\begin{aligned}
& \dot{x}_{1}=x_{2} \omega, \\
& \dot{x}_{2}=-x_{1} \omega-x_{2}\left(x_{1}^{2}+x_{2}^{2}-1\right)+u .
\end{aligned}
$$

Potential solutions of autonomous BO (22) in the limit cycle mode are:

$$
x_{1}(t)=-\cos (\omega t+\phi), x_{2}(t)=\sin (\omega t+\phi) .
$$

Solutions of the BO show that it can be used as an orthogonal signal generator. For a suitably designed control $u$, it is possible to ensure the synchronization of the $\mathrm{BO}$ output with that of the grid. In this context, equations of the BO-based frequency-locked loop (BO-FLL) as presented in [27, 28, 29, 30] are given by:

$$
\begin{aligned}
\dot{x}_{1} & =x_{2} \hat{\omega}, \\
\dot{x}_{2} & =-x_{1} \hat{\omega}-x_{2}\left(x_{1}^{2}+x_{2}^{2}-1\right)+\alpha\left(v-x_{2}-x_{3}\right) \hat{\omega}, \\
\dot{\hat{\omega}} & =-\beta x_{1}\left(v-x_{2}-x_{3}\right) \hat{\omega}, \\
\dot{x}_{3} & =\gamma\left(v-x_{2}-x_{3}\right) \hat{\omega},
\end{aligned}
$$

where $v$ is the grid voltage measurement from (21), $\alpha, \beta, \gamma>0$ are the positive tuning parameters, $\hat{\omega}$ is the estimated grid frequency and $x_{3}$ is an estimate of the DC offset. Using the state variables of the BO-FLL, the instantaneous phase of the grid voltage can be estimated as [30]:

$$
\hat{\Omega}=\bmod \left\{\operatorname{atan} 2\left(\frac{x_{2}}{-x_{1}}\right), 2 \pi\right\},
$$

where atan 2 denotes the double-quadrant arc-tangent.

\subsection{Experimental Study}

An experimental study is reported in this section to demonstrate the suitability of the BO-FLL for grid-synchronization applications. Hardware-in-the-loop (HIL) setup using dSPACE is used for this purpose. Parameters of the BO-FLL are chosen as: $\alpha=1.41, \beta=20$, and $\gamma=85$. Enhanced phase-locked loop (EPLL) [46] is selected as the comparison technique with parameters: $\mu_{0}=85$, $\mu_{1}=200, \mu_{2}=20 \times 10^{3}$, and $\mu_{3}=400$.

Two experimental cases are considered. In the first test, a $+5 \mathrm{~Hz}$ frequency step change is considered. Experimental results for this case are given in Fig. 


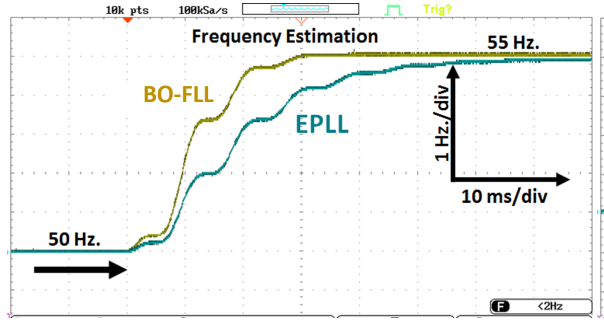

(a)

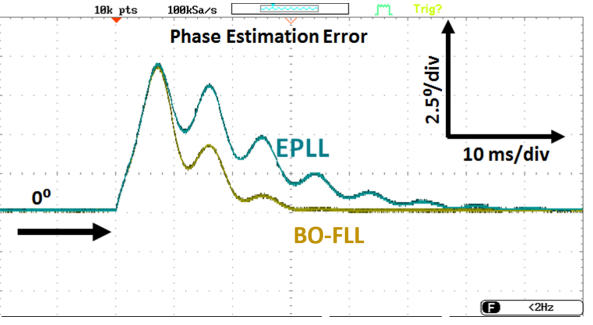

(b)

Figure 3: Experimental for a step frequency change of $+5 \mathrm{~Hz}$ : (a) Estimated frequency and (b) Phase estimation error.

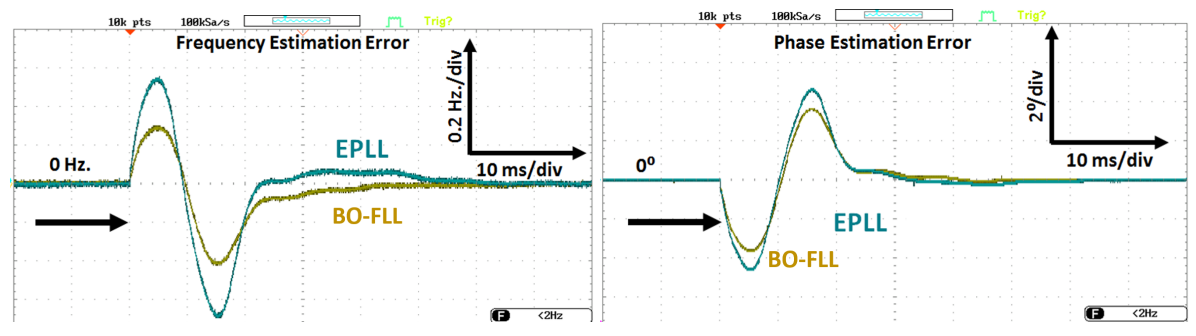

(a) (b)

Figure 4: Experimental for a step DC offset change of +0.1 .u.: (a) Estimated frequency and (b) Phase estimation error.

3. Results show that BO-FLL has a fast convergence with relatively smaller overshoot compared to EPLL. In the second test, suddenly, a DC offset of 0.1 per unit (normalized value with respect to the nominal amplitude) is added to the voltage, and experimental results for this case are given in Fig. 4. The frequency estimated by BO-FLL converged within $\approx 2$ cycles while it took slightly more time for EPLL. The error magnitudes are also smaller for BO-FLL compared to EPLL for both frequency and phase estimation error. These results show that BO-FLL has excellent potential to be used as a grid-synchronization tool.

\section{Conclusion and Outlook}

Some recent results on the robust synchronization of heterogeneous nonlinear systems, as presented in [1], are summarized in this paper. In addition, an application example is also presented involving the grid-synchronization of renewable energy sources. Heterogeneity and nonlinearity are two commonly available features of many systems of practical interest. As such, the presented results are very promising to further research in this area.

The analytical results considered mainly the cycle-graph for network interconnection among the agents. In the future, it would be interesting to investigate the effect of various other network topologies. Network-induced delays 
are often unavoidable for practical systems. As such, studying the delay (homogeneous and/or heterogeneous) robustness of the synchronization protocol could be another potential research direction. A significant limitation of the presented results is that they consider the systems to be robustly stable, a condition that cannot always be guaranteed. Relaxing this condition would enlarge the type of nonlinear systems for which synchronization results could be further developed. In developing the synchronization protocol for Brockett oscillators, linear feedback is used; as a future research direction, nonlinear feedback can be considered.

From the application point of view, only a grid-following converter is studied for synchronization purposes. The ever-increasing presence of renewable energy sources has made grid-forming converter $[47,48]$ an active research topic. In this context, virtual oscillator controls have attracted some consideration in recent times. Extending the summarized results to this new type of converters could also be considered as an inspiring research direction.

\section{References}

[1] H. Ahmed, Modeling and synchronization of biological rhythms: from cells to oyster behavior, Phd thesis (received the 2019 European PhD Award on Control), University of Lille (2016).

[2] S. H. Strogatz, Nonlinear dynamics and chaos: With applications to physics, biology, chemistry, and engineering, CRC press, 2018.

[3] F. Blaabjerg, R. Teodorescu, M. Liserre, A. V. Timbus, Overview of control and grid synchronization for distributed power generation systems, IEEE Transactions on industrial electronics 53 (5) (2006) 1398-1409.

[4] K. Horikawa, K. Ishimatsu, E. Yoshimoto, S. Kondo, H. Takeda, Noiseresistant and synchronized oscillation of the segmentation clock, Nature 441 (7094) (2006) 719-723.

[5] M. Feki, An adaptive chaos synchronization scheme applied to secure communication, Chaos, Solitons \& Fractals 18 (1) (2003) 141-148.

[6] R. Martinez-Guerra, W. Yu, Chaotic synchronization and secure communication via sliding-mode observer, International Journal of Bifurcation and Chaos 18 (01) (2008) 235-243.

[7] I. Skog, P. Handel, Time synchronization errors in loosely coupled GPSaided inertial navigation systems, IEEE transactions on intelligent transportation systems 12 (4) (2011) 1014-1023.

[8] M. Sen, C. S. L. Cajún, Review of synchronization in mechanical systems, in: Nonlinear Structural Dynamics and Damping, Springer, 2019, pp. 4570 . 
[9] Y. Tang, F. Qian, H. Gao, J. Kurths, Synchronization in complex networks and its application-a survey of recent advances and challenges, Annual Reviews in Control 38 (2) (2014) 184-198.

[10] M. Maghenem, R. Postoyan, A. Loria, E. Panteley, Lyapunov-based synchronization of networked systems: From continuous-time to hybrid dynamics, Annual Reviews in Control (2020).

[11] R. Sepulchre, Consensus on nonlinear spaces, Annual reviews in control 35 (1) (2011) 56-64.

[12] H. Zhang, F. L. Lewis, A. Das, Optimal design for synchronization of cooperative systems: state feedback, observer and output feedback, IEEE Transactions on Automatic Control 56 (8) (2011) 1948-1952.

[13] L. Scardovi, R. Sepulchre, Synchronization in networks of identical linear systems, in: 2008 47th IEEE Conference on Decision and Control, IEEE, 2008, pp. 546-551.

[14] P. Wieland, R. Sepulchre, F. Allgöwer, An internal model principle is necessary and sufficient for linear output synchronization, Automatica 47 (5) (2011) 1068-1074.

[15] A. Isidori, L. Marconi, G. Casadei, Robust output synchronization of a network of heterogeneous nonlinear agents via nonlinear regulation theory, IEEE Transactions on Automatic Control 59 (10) (2014) 2680-2691.

[16] A. Bidram, F. L. Lewis, A. Davoudi, Synchronization of nonlinear heterogeneous cooperative systems using input-output feedback linearization, Automatica 50 (10) (2014) 2578-2585.

[17] H. Modares, F. L. Lewis, W. Kang, A. Davoudi, Optimal synchronization of heterogeneous nonlinear systems with unknown dynamics, IEEE Transactions on Automatic Control 63 (1) (2017) 117-131.

[18] T. Hatanaka, N. Chopra, M. Fujita, M. W. Spong, Passivity-based control and estimation in networked robotics, Springer, 2015.

[19] A. V. Proskurnikov, M. Mazo Jr, Simple synchronization protocols for heterogeneous networks: beyond passivity, IFAC-PapersOnLine 50 (1) (2017) 9426-9431.

[20] A. Selivanov, A. Fradkov, E. Fridman, Passification-based decentralized adaptive synchronization of dynamical networks with time-varying delays, Journal of the Franklin Institute 352 (1) (2015) 52-72.

[21] H. Ahmed, R. Ushirobira, D. Efimov, W. Perruquetti, Robust synchronization for multistable systems, IEEE Transactions on Automatic Control 61 (6) (2016) 1625-1630. 
[22] H. Ahmed, R. Ushirobira, D. Efimov, Robust global synchronization of Brockett oscillators, IEEE Transactions on Control of Network Systems 6 (1) (2019) 289-298.

[23] R. Brockett, Synchronization without periodicity, Mathematical systems theory, a volume in honor of U. Helmke (2013) 65-74.

[24] H. Ahmed, R. Ushirobira, D. Efimov, On the robust synchronization of Brockett oscillators, IFAC-PapersOnLine 49 (14) (2016) 142-147.

[25] H. Ahmed, R. Ushirobira, D. Efimov, Experimental study of the robust global synchronization of Brockett oscillators, The European Physical Journal Special Topics 226 (15) (2017) 3199-3210.

[26] H. Ahmed, R. Ushirobira, D. Efimov, L. Fridman, Y. Wang, Output global oscillatory synchronization of heterogeneous systems, International Journal of Control (2019) 1-21.

[27] S. Biricik, H. Komurcugil, H. Ahmed, E. Babaei, Super twisting sliding mode control of DVR with frequency-adaptive Brockett oscillator, IEEE Transactions on Industrial Electronics (2020) 1-1.

[28] H. Ahmed, M. Bierhoff, M. Benbouzid, Multiple nonlinear harmonic oscillator-based frequency estimation for distorted grid voltage, IEEE Transactions on Instrumentation and Measurement 69 (6) (2020) 2817 2825 .

[29] M. L. Pay, H. Ahmed, Modeling and tuning of circular limit cycle oscillator FLL with preloop filter, IEEE Transactions on Industrial Electronics 66 (12) (2019) 9632-9635.

[30] H. Ahmed, S. Amamra, M. Bierhoff, Frequency-locked loop-based estimation of single-phase grid voltage parameters, IEEE Transactions on Industrial Electronics 66 (11) (2019) 8856-8859.

[31] D. Angeli, D. Efimov, Characterizations of input-to-state stability for systems with multiple invariant sets, IEEE Transactions on Automatic Control 60 (12) (2015) 3242-3256.

[32] D. Angeli, D. Efimov, On input-to-state stability with respect to decomposable invariant sets, in: 52nd IEEE Conference on Decision and Control, IEEE, 2013, pp. 5897-5902.

[33] Z. Nitecki, M. Shub, Filtrations, decompositions, and explosions, American Journal of Mathematics 97 (4) (1975) 1029-1047.

[34] J. Guckenheimer, P. Holmes, Structurally stable heteroclinic cycles, in: Mathematical Proceedings of the Cambridge Philosophical Society, Vol. 103, Cambridge University Press, 1988, pp. 189-192. 
[35] P. Forni, D. Angeli, The ISS approach to the stability and robustness properties of nonautonomous systems with decomposable invariant sets: An overview, European Journal of Control 30 (2016) 50-60.

[36] P. Monzón, R. Potrie, Local and global aspects of almost global stability, in: Proceedings of the 45th IEEE Conference on Decision and Control, IEEE, 2006, pp. 5120-5125.

[37] R. Marino, P. Tomei, Nonlinear Control Design: Geometric, Adaptive and Robust, Prentice-Hall, 1995.

[38] S. Dashkovskiy, D. V. Efimov, E. D. Sontag, Input to state stability and allied system properties, Automation and Remote Control 72 (8) (2011) 1579 .

[39] L. Fridman, Y. Shtessel, C. Edwards, X.-G. Yan, Higher-order sliding-mode observer for state estimation and input reconstruction in nonlinear systems, International Journal of Robust and Nonlinear Control 18 (4-5) (2008) 399412.

[40] A. Levant, Higher-order sliding modes, differentiation and output-feedback control, International journal of Control 76 (9-10) (2003) 924-941.

[41] A. F. Filippov, Differential equations with discontinuous righthand sides: control systems, Vol. 18, Springer Science \& Business Media, 2013.

[42] M. Krstic, P. V. Kokotovic, I. Kanellakopoulos, Nonlinear and adaptive control design, John Wiley \& Sons, Inc., 1995.

[43] H. Ahmed, S.-A. Amamra, I. Salgado, Fast estimation of phase and frequency for single-phase grid signal, IEEE Transactions on Industrial Electronics 66 (8) (2019) 6408-6411.

[44] H. Ahmed, S. Biricik, M. Benbouzid, Enhanced frequency adaptive demodulation technique for grid-connected converters, IEEE Transactions on Industrial Electronics (2020).

[45] H. Ahmed, I. Salgado, I. Chairez, M. Benbouzid, Robust gradient estimator for unknown frequency estimation in noisy environment: Application to grid-synchronization, IEEE Access 8 (2020) 70693-70702.

[46] M. Karimi-Ghartemani, S. A. Khajehoddin, P. K. Jain, A. Bakhshai, M. Mojiri, Addressing DC component in PLL and notch filter algorithms, IEEE Transactions on Power Electronics 27 (1) (2011) 78-86.

[47] T. Qoria, Grid-forming control to achieve a $100 \%$ power electronics interfaced power transmission systems, Ph.D. thesis, HESAM Université (2020).

[48] T. Qoria, F. Gruson, F. Colas, X. Kestelyn, X. Guillaud, Current limiting algorithms and transient stability analysis of grid-forming VSCs, Electric Power Systems Research 189 (2020) 106726. 


\section{Declaration of interests}

$\bigotimes$ The authors declare that they have no known competing financial interests or personal relationships that could have appeared to influence the work reported in this paper.

$\square$ The authors declare the following financial interests/personal relationships which may be considered as potential competing interests:

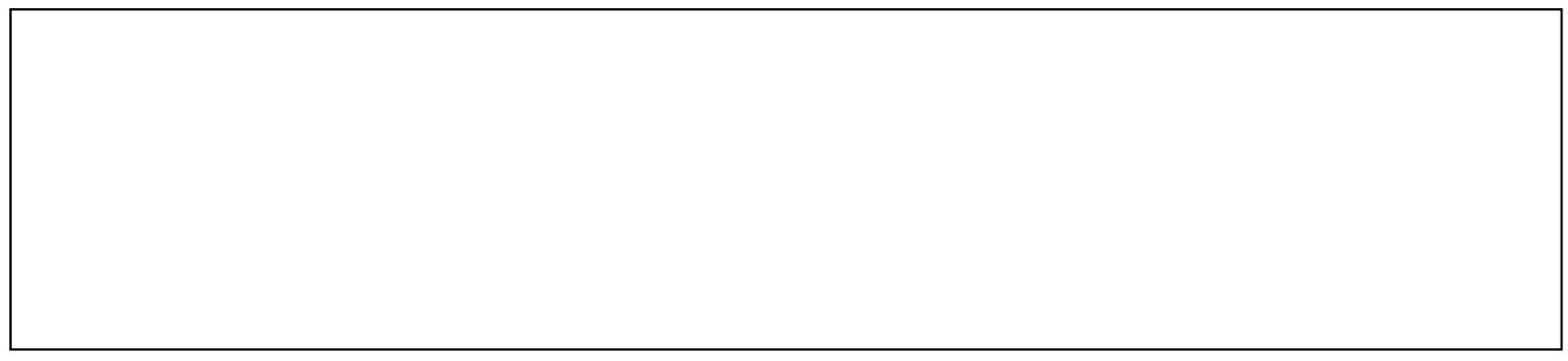

